\title{
Development of Disseminated Tuberculosis in A Non-Immunocompromised Patient After COVID-19 Infection: A Case Report
}

Ensiyeh Rahimi ( $\nabla$ ensiyehrahimi@gmail.com )

Tehran University of Medical Sciences https://orcid.org/0000-0002-3332-2378

\section{Sara Ghaderkhani}

Tehran University of Medical Sciences

Yousef Jamshidi

Shahid Beheshti University of Medical Sciences School of Pharmacy

\section{Case report}

Keywords: COVID-19, infection, TB, patient

Posted Date: July 26th, 2021

DOI: https://doi.org/10.21203/rs.3.rs-704284/v1

License: (c) (i) This work is licensed under a Creative Commons Attribution 4.0 International License.

Read Full License 


\section{Abstract}

\section{Background}

COVID-19 pandemic has had complications and has changed the pattern of disease incidence. COVID-19 affects the immune system. We present a case of disseminated tuberculosis (TB) in nonimmunocompromised person with a history of COVID-19 infection.

\section{Case presentation}

A 25 year old woman had a history of mild COVID-19 infection 3 months ago. Then the complaint of back pain began and the headache was added 1 months later. Gradually, the patient's headache intensified. Finally, the patient was admitted with decreased level of consciousness, delirium and focal neurological symptoms. The work up confirmed disseminated TB contain of miliary pulmonary TB, TB meningitis and TB spondylodiscitis. The patient gradually improved on anti-tuberculosis treatment.

\section{Conclusions}

There may be an association between disseminated TB infection following COVID-19 infection.

\section{Background}

Tuberculosis is a contagious disease that is one of the top 10 causes of death worldwide and the leading cause of death from a single infectious agent. In 2019, about 10 million people developed TB and 1.4 million died[ $\left.{ }^{1}\right]$.

MTB is transmitted from person to person via the airborne route[ $\left.{ }^{2}\right]$. After inhalation, the droplet nucleus is carried down the bronchial tree and implants in a respiratory bronchiole or alveolus. Whether or not inhaled tubercle bacilli establish an infection depends on both host and microbial factors $\left[{ }^{3}\right]$. Miliary TB is a type of disseminate TB that results from lymphatic and hematologic spread from a TB focus and is very fatal if not treated in time $\left[{ }^{4}\right]$. In countries with low incidence, delayed diagnosis and treatment, increase mortality $\left[{ }^{5}\right]$.

COVID-19 pandemic has had complications and has changed the pattern of disease incidence. COVID-19 affects the immune system. TB reactivation can be explained by T-cell depletion resulting from COVID-19 infection $[6,7,8]$. Here, we describe a patient who was diagnosed with the disseminated TB and history of COVID-19 infection.

\section{Case Presentation}

A 25-year-old Iranian female with complaints of headache and loss of consciousness was hospitalized on 2 Jun 2021. She presented with history of headache last 2 months. The patient's headaches were 
mostly in the frontal lobes and parietal lobes. She had frequent outpatient visits to the physician for headaches and has taken analgesics during this time.

The patient was non-immunocompromised person. She had a history of COVID-19 infection 3 months ago. Her COVID-19 disease was mild. She had flu-like syndrome and lost her sense of smell and taste. She didn't have COVID-19 pulmonary involvement and was not admitted to a hospital. Then the complaint of back pain began. The patient's back pain was on the thoracic vertebrae. The patient back pain has continued until now.

The patient's headache had worsened from 2 weeks before the admission. Finally, she came to the hospital with a severe headache. She had complaints of decreased level of consciousness, nausea, vomiting, diplopia, back pain and bladder incontinency. She had acute onset of lower limb weakness on day prior to the admission that become unable to even stand independently. Patient did not have any medical problem previously. She did not have any cough. There was no family history of TB. She has lost $5 \mathrm{~kg}$ in the last month. She had moved from village to city 22 years ago.

On admission, she was lethargic and confused. She had delirium. On physical examination she was afebrile. Signs of meningeal irritation were present. Lower limbs power decreased (4/5). She had nystagmus on eye examination.

Brain magnetic resonance images (MRI) showed diffused leptomeningeal enhancement. Mild hydrocephaly was seen too. There was abnormal signal area with restriction in splenium of corpus callosum without enhancement (Figure 1).

Spinal MRI showed abnormal signal intensity with enhancement in T8-T9 disc space, adjacent end plates, bodies and posterior elements and small part of body of T10 associated prevertebral soft tissue edema and enhancement (Figure 2).

On chest computed tomographic scan, poorly spread nodularity was seen throughout the lungs suggesting miliary tuberculosis. Multiple lymphadenopathies were seen in the left axillary and mediastinum that some of them had calcifications (Figure 3).

Laboratory investigations showed elevated WBC in Cerebral Spinal Fluid (60\% lymphocyte), high CSF protein and low glucose (Table 1). Mycobacterium tuberculosis detected with polymerase chain reaction (PCR) of cerebral spinal fluid (CSF). Rifampin resistance not detected with the Expert MTB/RIF assay. CMV, EBV, HSV1,2 and VZV didn't detect with PCR of CSF. White blood cell count was 5,900 cells $/ \mathrm{mm} 3$, ( $90 \%$ neutrophils, $8 \%$ lymphocytes). C-reactive protein level was $21 \mathrm{mg} / \mathrm{dl}$. Erythrocyte sedimentation rate (ESR) level was $31 \mathrm{~mm} / \mathrm{h}$. HIV serology was negative. Liver function tests parameters were containing: Aspartate transaminase (AST) $57 \mathrm{U} / \mathrm{L}$, Alanine transaminase (ALT) $53 \mathrm{U} / \mathrm{L}$, Alkaline phosphatase (ALP) $264 \mathrm{U} / \mathrm{L}$, serum bilirubin total $0.8 \mathrm{mg} / \mathrm{dl}$ and direct $0.5 \mathrm{mg} / \mathrm{dl}$. SARS CoV2 RT-PCR from nasopharyngeal and throat swabs was negative. 
Patient started on anti-tuberculosis drugs and steroid. She was started on isoniazid $300 \mathrm{mg}$, rifampin $600 \mathrm{mg}$, pyrazinamide $1200 \mathrm{mg}$ and ethambutol $825 \mathrm{mg}$ once daily. According to objective neurological findings, prednisolone $80 \mathrm{mg} /$ day was required, which was gradually tapered and discontinued through 6 weeks. Analysis of Cerebral Spinal Fluid (CSF) on the eighth day of treatment was better. Post treatment showed significant neurological improvement. The patient's consciousness gradually improved during the first week of treatment. Nystagmus and diplopia was improved during the second week. The patient's headache subsided and was mostly after waking up during the third week. Delirium was completely gone in the third week. From the fourth week, force of her lower limbs improved and she was able to stand independently. Despite T8-T9 spondylodiscitis, the patient had not neurological deficits and cord compression. According to the neurosurgeon consulting, she did not need to surgery and was treated with medical treatment. The patient's liver function tests increased at the beginning of treatment but gradually decreased after 2 weeks of treatment (Table2). The increase in liver function tests was less than 5 fold normal and the patient did not show signs and symptoms of acute hepatitis, so the medication continued. On day 18th of admission, the patient was discharged home in good general condition. The patient was prescribed anti tuberculous treatment for total duration of 12 months with a follow up appointment at the TB clinic. She received prednisolone for first 6 weeks' duration and her prednisolone decreased weekly.

\begin{tabular}{|lll|}
\hline \multicolumn{2}{|l|}{ Table 1 Analysis of Cerebral Spinal Fluid (CSF) } \\
\hline Variable & CSF 1th Day & CSF 8th Day \\
\hline White blood cells (cells/ML) & 50 & 2 \\
Red blood cells (cells/ML) & 3 & 2 \\
Neutrophils (\%) & 40 & - \\
Lymphocyte (\%) & 60 & - \\
Glucose (mmol/L) CSF/G Serum & $39 / 109$ & $65 / 120$ \\
LDH (U/L) & 149 & 68 \\
Protein (g/L) & 130 & 19.5 \\
\hline
\end{tabular}

\begin{tabular}{|llllllllllll|}
\hline \multicolumn{10}{|c|}{ Table 2 Liver Function Tests during treatment } \\
\hline Day & 1th & 3th & 5 th & 7th & 9th & 11th & 13th & 15th & 17th & 19th \\
AST & 57 & 80 & 115 & 50 & 76 & 137 & 128 & 84 & 52 & 50 \\
ALT & 53 & 53 & 85 & 69 & 111 & 135 & 125 & 110 & 82 & 62 \\
\hline
\end{tabular}

\section{Discussion And Conclusions}


An interesting point in this case is the disseminated TB in a young woman with no history of the disease and only looking for a mild COVID-19 infection. The patient had a disseminated TB with military TB pulmonary, TB meningitis and TB spondylodiscitis. Our patient was not immunocompromised. She only had a history of mild COVID-19 infection. The patient received only a few outpatient doses of dexamethasone due to the non-hospitalization. The complaint of back pain began after COVID-19 infection. No underlying cause for her disseminated TB was found. There may be an association between disseminated TB infection following COVID-19 infection. Several studies have reported that CD4 T cells play an essential role in defense against MTB $\left[{ }^{9}\right]$. In Diao et al[7] study, 76\% COVID-19 infected patients had significant depletion in their T-cell lymphocyte counts. Both CD4 and CD8 counts were severely reduced, and the surviving $T$ cells appeared to be dysfunctional. This $T$ cell depletion and exhaustion may promote latent TB to active TB. In acute COVID-19 infection, peripheral lymphocyte especially T lymphocyte are depleted. CD8 T lymphocytes will be recovered within 2-3 months and CD4 T lymphocytes will return to normal within a year $\left.{ }^{10}\right]$.

Few studies have linked TB reactivation to viral infections $\left[8,{ }^{11}\right]$. Few studies have demonstrated the association between active TB and COVID-19 infection $[12,13,14,15]$ or a flare up of TB after COVID-19 infection $\left[{ }^{16},{ }^{17}\right]$. Tadolini et al[13] and Stochino et al[14] had reported patients with concurrent COVID-19 infection with active TB infection. In Tadolini[13] study coinfection TB an COVID-19 presented with median time of 4 days. They thought that the overlap of symptoms between the two diseases led to diagnosis of tuberculosis. With Elzny et al[8] reported a patient with possible peritoneal TB who progressed to miliary pulmonary TB after COVID-19 infection.

Most cases of tuberculosis have been seen after sever COVID-19 infection[ $\left.{ }^{18}\right]$ but in our study and Khayat M. et al[6] study, tuberculosis was looking for a mild COVID-19 infection.

In endemic areas of tuberculosis, it is important to pay attention to patient's symptoms after COVID-19 infection such as weakness, headache and weight loss. As this case has had weakness, headache, and weight loss for few months. These symptoms may be confused with post COVID-19 syndrome and lead to delays the diagnosis and treatment of tuberculosis.

Given that the COVID-19 infection is a risk factor for TB reactivation. In COVID-19 pandemic, early identification of TB patients is important for treatment and infection control.

Due to wide patient involvement, complete and timely treatment of the patient is important. The success of drug treatment, depends upon many factors. Some studies have found an increased risk of relapse among patients with signs of more extensive disease $\left[{ }^{19}\right]$.The patient was prescribed 12 months of antituberculosis treatment. Tuberculosis treatment requires multiple drugs be given for several months. It is difficult for the patient to tolerate the treatment due to the possibility of drug side effects. The patient should be aware of the importance of taking the medication correctly. It is very important that the patient be involved in a making decisions concerning treatment supervision and overall care. International 
standards also emphasize the importance of using patient centered approaches to the treatment of tuberculosis $\left[{ }^{20}\right]$.

\section{Declarations}

\section{Ethical approval and consent to participate}

This research was approved by the Ethics Committee of our hospital. The patient privacy is respected. Patient's name did not be used. Images of patients did not be used.

\section{Consent for publication}

Consent was obtained from the patient for publication of this case report and accompanying images. A copy of the written consent is available for review by the

\section{Editor of this journal.}

\section{Availability of data and materials}

Data sharing not applicable to this article as no datasets were generated or analyzed during the current study.

\section{Competing interests}

The authors do not have any competing interests.

\section{Funding}

No funding was received for this study.

\section{Author contributions}

E Rahimi: care of patient, supervision, writing, original draft, submitting. S Ghaderkhani: care of patient, editing. Y Jamshidi: consulting, writing, review and editing.

\section{Affiliations}

Department of infectious diseases, Imam Khomeini hospital, Iran, Tehran. Ensiyeh Rahimi Department of infectious diseases, Imam Khomeini hospital, Iran, Tehran. Sara Ghaderkhani Faculty of Pharmacy, Shahid Beheshti University of Medical Science, Tehran, Iran. Yousef Jamshidi

Corresponding author

Correspondence to Ensiyeh Rahimi. 
Acknowledgements

We thank the member of Department of Infectious Diseases, Imam Khomeini Hospital, Tehran University of Medical Science.

\section{References}

1. Moscow Declaration to End TB; First WHO global ministerial conference on ending TB in the sustainable development era: a multisectoral response. Geneva: World Health Organization and the Ministry of Health of the Russian Federation; 2017 (https://www.who.int/tb/features_archive/Moscow_Declaration_to_End_TB_final_ENGLISH.pdf? ua $=1$, accessed 28 June 2019).

2. Reichman LB, Hershfield ES; ebrary Inc.Tuberculosis: a comprehensive international approach,Lung biology in health and disease v 144. 2nd ed. New York: Dekker, 2000: xxviii, 898 p.

3. Schluger NW, Rom WN. The host immune response to tuberculosis. Am J Respir Crit Care Med 1998; 157:679-91.

4. Sharma S. K., Mohan A., Sharma A., and Mitra D. K., Miliary tuberculosis: new insights into an old disease.The Lancet Infectious Diseases, 2005, vol. 5, no. 7, pp. 415-430.

5. Kethireddy S., Light R. B., Mirzanejad Y.et al. Mycobacterium tuberculosis septic shock. CHEST, 2013, vol. 144, no. 2, pp. 474-482.

6. Khayat M. Fan H., Vali Y., COVID-19 promoting the development of active tuberculosis in a patient with latent tuberculosis infection: A case report. Respiratory Medicine Case Reports 32,2021 , 101344.

7. Diao B., Wang Ch.,.Tan Y, Chen X., Liu Y., Ning L., Chen L., et al, Reduction and functional exhaustion of T cells in patients with coronavirus disease 2019 , Front. Immunol,2020, 11, Doi: 10.3389/fimmu.2020.00827.

8. Elziny Moustafa M., Ghazy A., Elfert Khaled A., and Aboukamar M., Development of Miliary Pulmonary Tuberculosis in a Patient with Peritoneal Tuberculosis after COVID-19 Upper Respiratory Tract Infection., Am. J. Trop. Med. Hyg., 104(5), 2021, pp. 1792-1795

9. Behar SM., Antigen-Specific CD8+ T Cells and Protective Immunity to Tuberculosis. The New Paradigm of Immunity to Tuberculosis. 2013, pp. 141-163. Doi: 10.1007/978-1-4614-6111-1_8

10. Lin L., Lu L., Cao W., Li. T., Hypothesis for potential pathogenesis of SARS-CoV-2 infection-a review of immune changes in patients with viral pneumonia, Emerg. Microb. Infect. 9 (1), 2020, 727-732, Doi:10.1080/22221751.2020.1746199.

11. Moss AR, Hahn JA, Tulsky JP, Daley CL, Small PM, Hopewell PC, 2000. ,Tuberculosis in the homeless: a prospective study. , Am J Crit Care Med. Volume 162, Issue 2. Available at: Doi:

10.1164/ajrccm.162.2.9910055. Accessed February 5, 2021. 
12. Liu C, Yu Y, Fleming J, Wang T, Shen S, Wang Y, Fan L, Ma J, Gu Y, Chen Y, Severe COVID-19 cases with a history of active or latent tuberculosis. , Int J Tuberc Lung Dis, 2020. Jul 1;24(7):747-749. Doi:10.5588/ijtld.20.0163

13. Tadolini M., Codecasa LR, Garcia-Garcia J.M., Blanc F.X, Borisov S., et al. Active tuberculosis, sequelae and COVID- 19 co-infection: first cohort of 49 cases. ,Eur Respir, 2020, J 56, Doi: 10.1183/13993003.01398-2020.

14. Stochino C, Villa S, Zucchi P, Parravicini P, Gori A, Raviglione MC.,Clinical characteristics of COVID-19 and active tuberculosis co-infection in an Italian reference hospital. Eur Respir, 2020, J 56: 2001708.

15. Tham SM, Lim WY, Lee CK, Loh J, Premkumar A, Yan B, Kee A, Chai L, TambyahPA, Yan G, Four patients with COVID-19 and tuberculosis, Singapore, ,Emerg Infect Dis, 2020, 26, Doi: 10.3201/eid2611.202752.

16. Yao Z, Chen J, Wang Q, Liu W, Zhang Q, Nan J, et al. Three patients with COVID-19 and pulmonary tuberculosis, Wuhan, China, January-February 2020. Emerg Infect Dis, 2020, 26, Doi: 10.3201/eid2611.201536.

17. Faqihi F, Alharthy A, Noor AF, Balshi A, Balhamar A, Karakitsos D, COVID-19 in a patient with active tuberculosis: a rare case-report. Respir Med Case Rep,2020, 31: 101146.

18. Pozdnyakov A, Jin, M Bader A, Reactivation of Pulmonary Tuberculosis in a Patient With COVID-19 Case Report and Review of Literature, Infectious Diseases in Clinical Practice: April 26, 2021 Volume Publish Ahead of Print

19. Gillespie SH, Crook AM, McHugh TD, et al. Four-month moxifloxacin-based regimens for drugsensitive tuberculosis. N Engl J Med 2014; 371:1577-87.

20. Hopewell PC, Fair EL, Uplekar M.,Updating the International Standards for Tuberculosis Care. Entering the era of molecular diagnostics. Ann Am Thorac Soc 2014; 11:277-85.

\section{Figures}




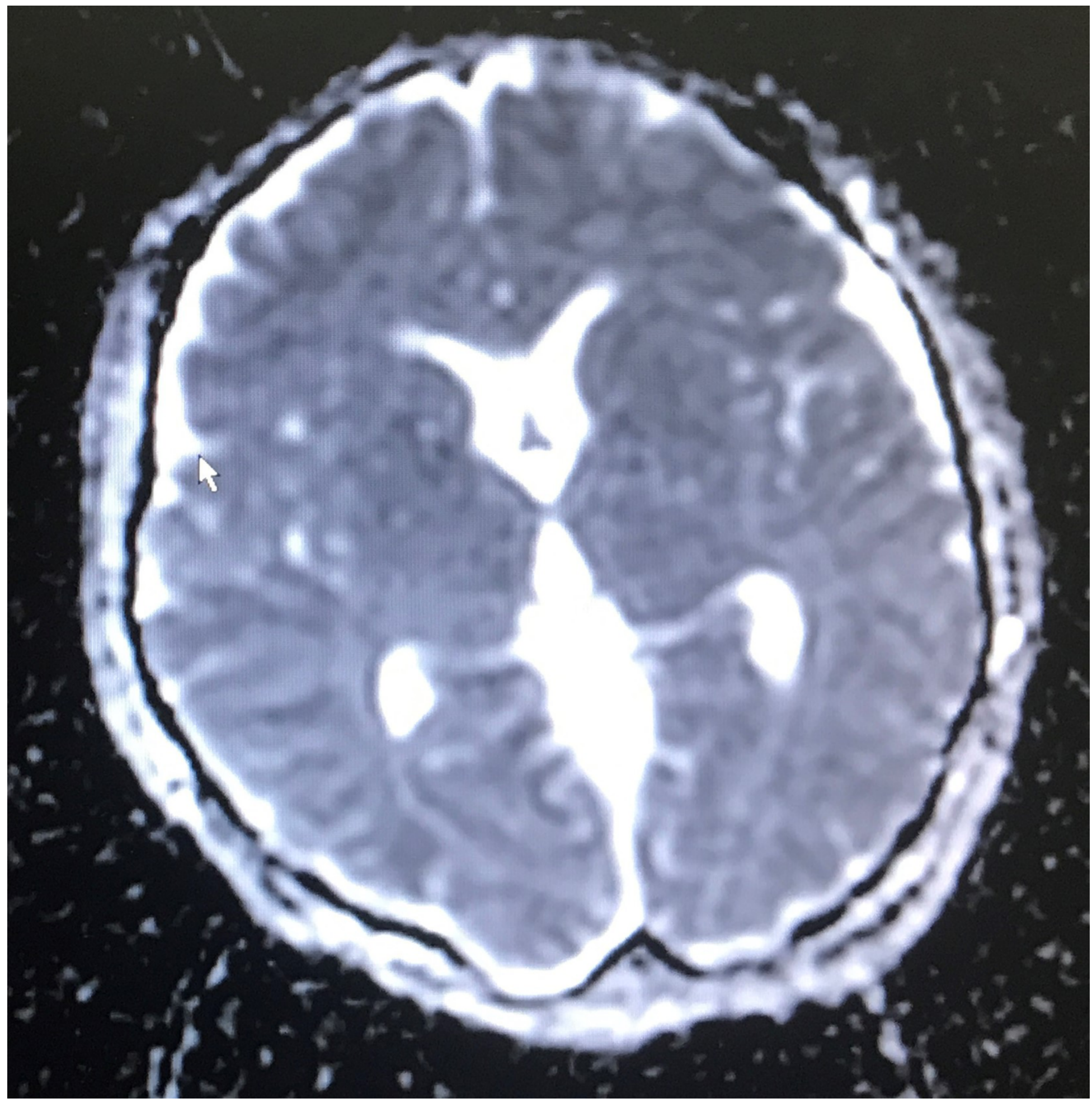

Figure 1

Brain MRI imaging shows diffused leptomeningeal enhancement 


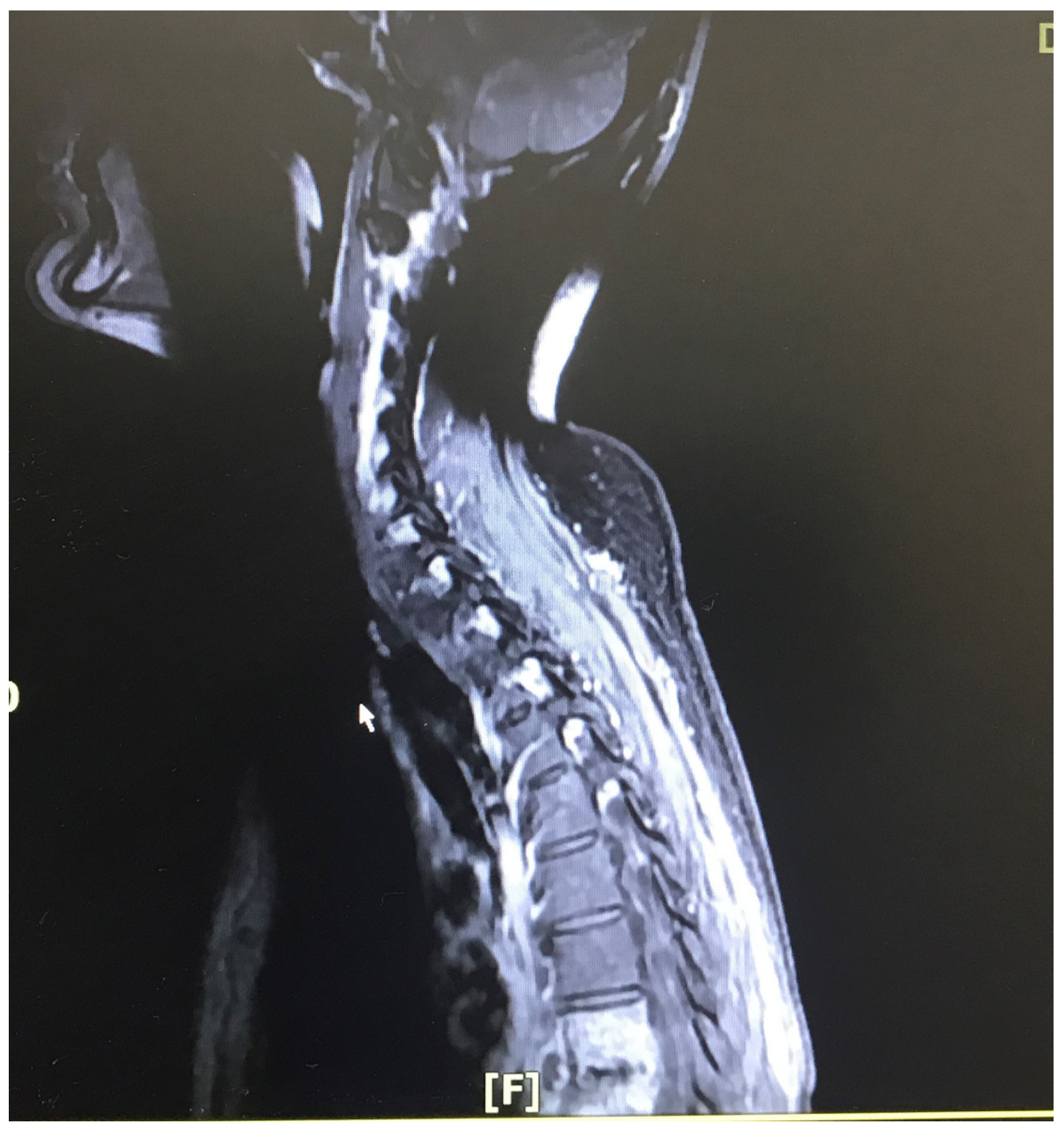

\section{Figure 2}

Spinal MRI imaging shows T8-T9 spondylodiscitis 


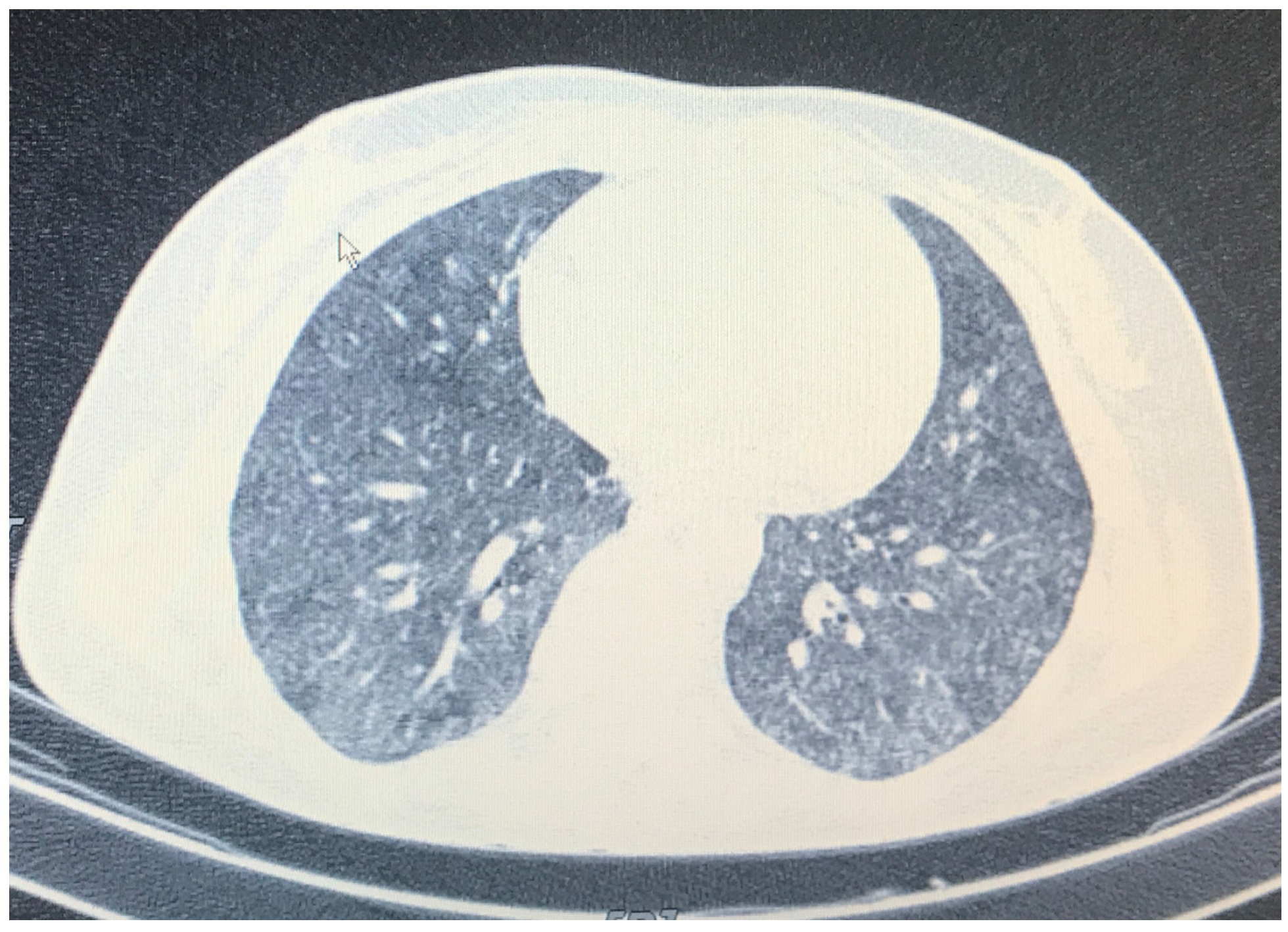

Figure 3

Chest Computed Tomographic Scan imaging shows lung miliary tuberculosis 